\title{
Steady-state organization of binary mixtures by active impurities
}

\section{Sabra, Mads Christian; Gilhøj, Henriette; Mouritsen, Ole G.}

\section{Published in:}

Physical Review E. Statistical, Nonlinear, and Soft Matter Physics

Link to article, DOI:

10.1103/PhysRevE.58.3547

Publication date:

1998

Document Version

Publisher's PDF, also known as Version of record

Link back to DTU Orbit

Citation (APA):

Sabra, M. C., Gilhøj, H., \& Mouritsen, O. G. (1998). Steady-state organization of binary mixtures by active impurities. Physical Review E. Statistical, Nonlinear, and Soft Matter Physics, 58(3), 3547-3551.

https://doi.org/10.1103/PhysRevE.58.3547

\section{General rights}

Copyright and moral rights for the publications made accessible in the public portal are retained by the authors and/or other copyright owners and it is a condition of accessing publications that users recognise and abide by the legal requirements associated with these rights.

- Users may download and print one copy of any publication from the public portal for the purpose of private study or research.

- You may not further distribute the material or use it for any profit-making activity or commercial gain

- You may freely distribute the URL identifying the publication in the public portal 


\title{
Steady-state organization of binary mixtures by active impurities
}

\author{
Mads C. Sabra, Henriette Gilhøj, and Ole G. Mouritsen* \\ Department of Chemistry, Technical University of Denmark, Building 206, DK-2800 Lyngby, Denmark
}

(Received 10 September 1997; revised manuscript received 20 January 1998)

\begin{abstract}
The structural reorganization of a phase-separated binary mixture in the presence of an annealed dilution of active impurities is studied by computer-simulation techniques via a simple two-dimensional lattice-gas model. The impurities, each of which has two internal states with different affinity for the two species, become active by an external driving of a transition between the two impurity states, leading to an energy flow from the impurities into the binary mixture. In steady state, the drive is found to break down the phase-separated state and lead to a new finite length scale controlled by the drive. The model can be extended to describe compartmentalization of biomembranes by active membrane-bound proteins. [S1063-651X(98)00809-5]

PACS number(s): 87.22.Bt, 05.70.Ln, 64.60.Cn, 64.75.+g
\end{abstract}

Phase-separation and spinodal decomposition processes in multicomponent mixed systems serve to organize the mixtures in space and time. For example, after a quench of a binary mixture from the disordered, fully mixed state to a temperature within the phase-coexistence region, the two components start separating. The separation proceeds via a nonequilibrium, time-dependent domain pattern characterized by dynamical scaling invariance of the structure factor and a length scale that usually diverges as a power law in time with an exponent whose value is determined by the conservation laws in effect [1]. Eventually, as the system approaches equilibrium, the two components have separated globally into two macroscopic, thermodynamic phases. The spontaneous nonequilibrium organization of the binary system at any given time is controlled by the excess free energy of the interfaces bounding the domains. The question now arises as to is how to avoid global separation and maintain some small-scale equilibrium or steady-state organization of the mixture, e.g., microphase separation, that is characterized by a finite length scale that does not change with time. Such an organization or compartmentalization of the binary mixture can be obtained by stabilizing the interfaces, using some suitable agent or process that lowers the interfacial free energy (tension). A well-known example is the use of surfaceactive agents to stabilize emulsions of liquid mixtures, e.g., surfactants such as soaps and cosurfactants such as alcohols in microemulsions of oil and water [2], or block-copolymers in immicible polymer blends [3]. Another possibility is to couple a chemical reaction to the interface dynamics that stabilizes the interface and induces a new, finite length scale into the problem [4-12].

In the present paper we shall consider a third possibility for a principle of compartmentalizing binary mixtures by introducing an annealed dilution of active impurities, which, by internal conversions between different states induced by external energy sources, couple to the interface dynamics. This coupling impedes the phase-separation process and leads to a steady-state structure characterized by a length scale that is determined by the strength of the external drive. We shall study the properties of this steady state using the

\footnotetext{
*Author to whom correspondence should be addressed. FAX: +45-45-934808. Electronic address: ogm@kemi.dtu.dk
}

simplest possible model, which is a two-dimensional latticegas model (Ising model with conserved order parameter) with an annealed dilution of active site impurities. The study was inspired by the possible application of this principle to steady-state compartmentalization of biological membranes. In these systems, the role of the active impurity is played by integral membrane proteins, which may change conformational state and hence change coupling to the lipid-bilayer part of the membrane $[13,14]$, due to external fields, e.g. photons in the case of light-sensitive proteins.

The lattice-gas model in its simplest version is described by the spin-1/2 Ising Hamiltonian

$$
\mathcal{H}=-\frac{J}{2} \sum_{i \in \Omega_{L}, \Omega_{P}} \sum_{j}^{\mathrm{NN}} \sigma_{i} \sigma_{j}
$$

where $\sigma_{i}= \pm 1$. The second sum is restricted to run over nearest neighbors to $i$ on a triangular lattice with periodic boundary conditions. The $N$ sites of the lattice have been divided corresponding to two sets of indices $\Omega_{L}$ and $\Omega_{P} . \Omega_{L}$ labels the two major species, the actual type of the species on site $i$ in question being determined by the sign of $\sigma_{i}$. In the same way, $\Omega_{P}$ labels the impurities, and the corresponding values of $\sigma_{i}$ determine the internal states of the impurities. The relation between the actual lattice sites and the division into the two sets is a dynamic one corresponding to an annealed mixture. All interactions are described by a single positive interaction constant $J$ corresponding to ferromagnetic (attractive) interactions. Hence, an impurity has an affinity for the type of spin species to which its spin state corresponds. The concentrations of the two major species and the impurities are taken to be conserved. With these definitions, Eq. (1) is nothing but a simple spin-1/2 Ising model in which a fixed and identifiable set of the spins, called the impurities, is labeled to be prone to activity. It is only when the activity is turned on that the model is different from a conserved spin-1/2 Ising ferromagnet. In contrast to many other studies of dilute and impure Ising models, the impurities in the present model is not spin-zero particles. Instead, they have the same spin states as the other spins; only their dynamics is different as described below.

The dynamics of the system is controlled by nearestneighbor Kawasaki particle exchange between any particle pair (spins or impurities) and the acceptance criterion for a 
move is given by the standard Monte Carlo Metropolis rate $\min \left\{1, \exp \left(-\Delta \mathcal{H} / k_{B} T\right)\right\}$. For simplicity, all pair exchanges are taken to occur on the same time scale $\tau_{0} \quad\left(\tau_{0}=1\right.$ in units of Monte Carlo steps per lattice site), i.e., the two major species and the impurities have the same diffusion constant. The impurities are made active by subjecting them at random to an internal transition $\sigma_{i} \rightarrow-\sigma_{i}$, using temperatureindependent spin-flip dynamics that does not conserve the magnetization. This internal change, which can be imagined to take place due to an external drive, occurs at a time scale $\tau$ (in units of $\tau_{0}$ ). The strength of the drive can therefore conveniently be described by a parameter $\Gamma=\tau^{-1}$. Because each site of the lattice is coupled to the heat bath, there is no conservation of energy. Hence the dissipation of energy in the system takes place under isothermal conditions. This is a realistic condition for, e.g., lipid-bilayer membranes in water to be discussed below, where the thermocouple between the ultrathin membrane and the water phase is very fast. In the limit where all spins are prone for internal conversions, the model reduces to one previously studied by Glotzer and Coniglio [5].

In order to avoid complications due to percolation, most of the calculations to be reported below have been done for noncritical mixtures, i.e., compositions of plus and minus spins different from 1:1. Specifically we have considered a mixture ratio of 7:3 at an impurity concentration of $20 \%$. Other compositions have also been considered. A series of different lattice sizes have been studied in order to assess finite-size effects. The system is driven into a steady state by allowing it to relax from the initial state, in the presence of the drive, to a state where its energy, magnetization, and length scale (to be defined below) no longer change in time but only fluctuate around their steady-state values. In contrast to the work by Glotzer and Coniglio [5], here we have focused only on the steady state and not the time evolution of the system towards this state, e.g., subsequent to a thermal quench. The steady-state value of a property is obtained by averaging over a large number of configurations (typically $10^{5}$ ) characteristic of the steady state. The steady state is independent of the initial state and the system properties in the steady state are hence subject to self-averaging, in contrast to systems undergoing spinodal decomposition processes.

Shown in Figs. 1(a)-1(f) is a selection of microconfigurations typical of a steady state for a number of different driving strengths at two different temperatures $k_{B} T / J=1.5$ and 3.0, which are well within the equilibrium coexistence region. The figure shows qualitatively that in the presence of the drive, the equilibrium phase-separated state [Figs. 1(a) and 1(d)] breaks up and a steady-state domain pattern, reminiscent of a finite-time nonequilibrium pattern during a spinodal decomposition process, establishes itself on a length scale that depends on the drive. The interfaces are more rough at the higher temperature, due to the lower interfacial tension. The actual pattern is a dynamic one and it changes and fluctuates persistently in the steady state. These changes are faster the higher the temperature.

We have derived two independent quantitative measures of the steady-state length scale $R(\Gamma)$. The first measure is derived from the excess internal energy $\Delta E(\Gamma)=\mid E(T)$ $-E(\Gamma) \mid$, where $E(T)$ is the equilibrium energy correspond- ing to the globally phase-separated state and $E(\Gamma)$ is the energy in the steady state. Provided the width of the domain interfaces is small compared to the actual size of the domains, $\Delta E(\Gamma)$ is expected to scale as the total perimeter of the interfaces and hence to behave as a reciprocal length scale $\Delta E(\Gamma) \sim R_{E}^{-1}(\Gamma)[15]$. The second measure of the length scale that characterizes the domain pattern can be obtained as the average linear domain size $R_{D}$ calculated from the domain-size distribution. The domain-size distribution function in turn is calculated by direct evaluation of the domain sizes in a large ensemble of configurations typical of the steady state. Shown in Figs. 2(a) and 2(b) are, in a double-logarithmic plot, the results for $R_{E}(\Gamma)$ and $R_{D}(\Gamma)$ over a range of five orders of magnitude of the drive. Both data sets are seen to exhibit a systematic size dependence that is negligible for strong drives and significant for weak drives. For decreasing values of $\Gamma$, a finite system contains only a few domains, ultimately a single domain whose size is determined by the number of minority spins. For the largest system size studied, both data sets seem to have converged to a well-defined limit, with the exception of the weakest drives. This limit varies the same way with $\Gamma$ for both $R_{E}(\Gamma)$ and $R_{D}(\Gamma)$.

At low and intermediate values of the drive $\Gamma \leqslant 3$ $\times 10^{-1}, R_{E}(\Gamma)$ and $R_{D}(\Gamma)$ appear to display power-law scaling which we shall return to below. At large $\Gamma$ there is a flattening out of the curves and a slow crossover to a regime of drives that are so fast that the mixture starts sensing only an average state of the impurities. In the limit $\Gamma \rightarrow \infty$ (not studied here), the driven impurities do not have time to diffuse into the ordered domains and adapt to the domain structure before they flip again. Hence, as this limit is approached, the impurities have an increasing tendency to congregate at the domain interfaces. Eventually, a phase-separated state is reestablished provided the impurity concentration is not too high and the temperature is sufficiently low. Therefore, in this limit the present model has a different behavior from the model studied by Glotzer and Coniglio [5] that has a disordered phase for $\Gamma \rightarrow \infty$.

Theoretical analyses based on a self-consistent version of the Cahn-Hilliard equation for spinodal decomposition [5], appropriately modified to account for the suppression of the long-wavelength instability due to a chemical reaction in a binary system, have suggested that the time-dependent length scale varies as $R(t) \sim t^{p} F(t \Gamma)$, where $F$ is a scaling function with the property $F(x) \sim x^{-p}$ for $x \gg 1, p$ is a kinetic exponent, and $\Gamma$ plays the role of the rate of the chemical reaction. In the steady-state limit $t \rightarrow \infty$, the length scale $R$ will then scale with $\Gamma$ as a power law

$$
R(\Gamma) \sim \Gamma^{-p} .
$$

The kinetic exponent is predicted for low rates to be equivalent to the growth exponent of spinodal decomposition $p$ $=1 / 3$, whereas there is a crossover to $p=1 / 4$ for high rates $[6,10]$. These results were supported by numerical simulations of the modified Cahn-Hilliard equation $[6,10]$, whereas Monte Carlo simulations by Glotzer et al. [4] on the reactive binary mixture (Ising-type) model found only $p \sim 0.20$ -0.25 . A full molecular dynamics simulation on a reactive Lennard-Jones mixture led to similarly low effective expo- 

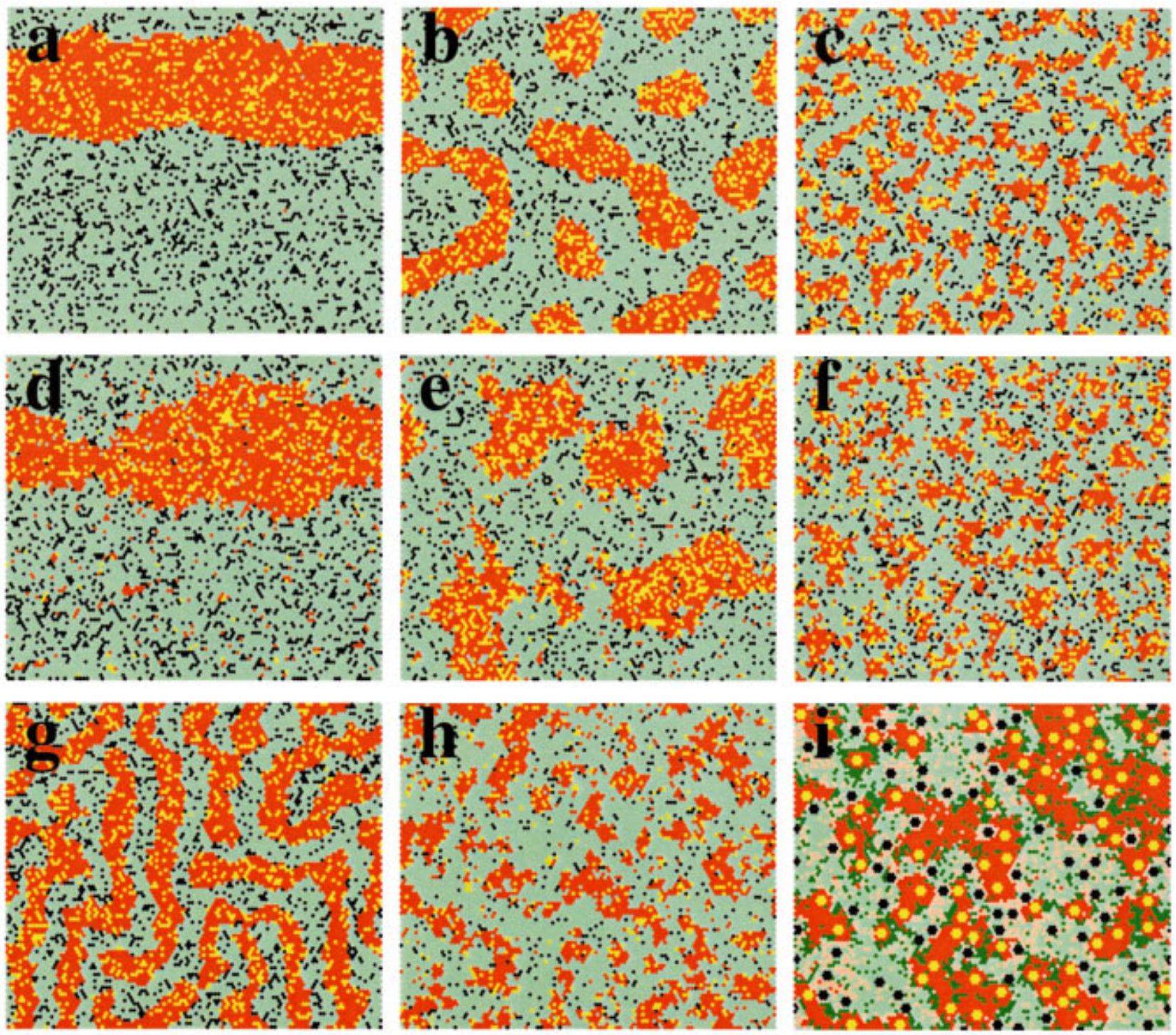

FIG. 1. (Color) Snapshot of steady-state domain organization of systems with active impurities. (a) -(c) The lattice-gas model at a composition $7: 3$ with $20 \%$ active impurities for different values of the driving strength $\Gamma=0,10^{-4}$, and $10^{-2}$ (from left to right). The temperature is $k_{B} T / J=1.5$. The two species are indicated in red and green and impurities in the corresponding spin states are shown in yellow and black, respectively. (d)-(f) Same as (a)-(c) at a temperature $k_{B} T / J=3$. (g) The lattice-gas model with $20 \%$ active impurities at a composition $1: 1, k_{B} T / J=1.5$, and $\Gamma=10^{-3}$. (h) Same as (f) with $10 \%$ active impurities. (i) The binary lipid membrane model at composition $1: 1$ with $10.5 \%$ (surface coverage) active seven-site proteins at a drive $\Gamma=10^{-3}$, and at a temperature inside the equilibrium coexistence region. The two species are colored in red and green and the degree of coloring reflects the internal conformational order of the acyl chains. Proteins are shown as yellow and black hexagons corresponding to the two internal protein states. The lattice size is in all cases $100 \times 100$ with toroidal periodic boundary conditions. The simulation unit cell, which has edges along the canonical axes of the triangular lattice, is folded onto a rectangular shape in order to save space. The boundary conditions therefore appear as staggered in the vertical direction.

nent values [7]. The results in Figs. 2(a) and 2(b) for $R(\Gamma)$ in the case of our binary mixture model with active impurities, in which the drive plays a role equivalent to the chemical reaction rate, support a power-law relation in an extended region of $\Gamma$ and are consistent with a gradual crossover from an effective exponent value around $p=1 / 3$ for weak drives to a somewhat smaller value around $p=1 / 4$ for stronger drives. It is to be expected in the scaling regime that the model studied in the present paper should behave in the same way as the model studied by Glotzer and Coniglio [5] in which all spins may be activated.

The scaling behavior is found to be fairly robust to the various parameters entering the model. Specifically, we have found similar results for compositions 1:1 and 3:2. The scaling behavior is moreover insensitive to temperature as illustrated in Fig. 2(c), although the crossover regime seems to move down with temperature. Furthermore, we have found that Hamiltonians more complex than Eq. (1), where specific repulsive or attractive interactions are assumed between the impurities, lead to the same scaling behavior for $R(\Gamma)$ as shown in Fig. 2. However, the transient behavior and the actual domain size and morphology will depend on such details. An example is shown in Fig. 1(g) of a steady-state pattern at a composition 1:1 where a very stringy and perco- 


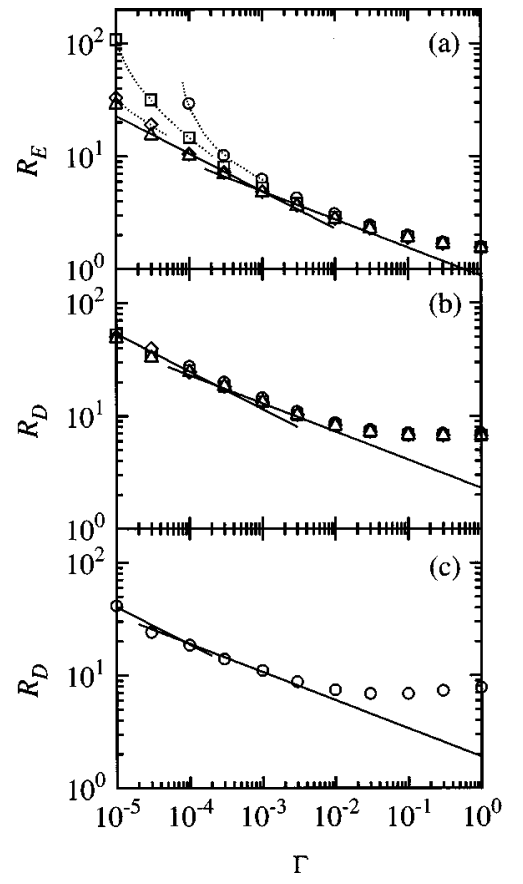

FIG. 2. Length-scale measures (a) $R_{E}(\Gamma)$ and (b) $R_{D}(\Gamma)$ for the lattice-gas model with active impurities subject to a drive of strength $\Gamma$ at a temperature $k_{B} T / J=3$. Data are shown for different systems sizes: $50 \times 50(\bigcirc), 100 \times 100(\square), 150 \times 150(\diamond)$, and $200 \times 200(\triangle)$. The lower panel (c) shows data for $R_{D}(\Gamma)$ at a lower temperature $k_{B} T / J=1.5$ and for a system of size $100 \times 100$. The solid lines have slopes $1 / 3$ and $1 / 4$ and are shown as guides to the eye. The dashed lines emphasize the systematics in the finitesize effects for weak drives.

lative labyrinthine pattern is seen to arise. The effect of lowering the impurity concentration is illustrated in Fig. 1(h).

Having established numerically, in the simplest possible setting, that an annealed dispersion of active impurities can lead to a steady-state spatial domain organization of a binary mixture, we now indicate how this principle can be implemented and used within a model of a specific system, a binary lipid-bilayer membrane incorporated with active transmembrane amphiphilic proteins [16]. A convenient model for a binary lipid-bilayer mixture is the ten-state lattice-gas model previously developed to describe the phase equilibria in mixtures of lipids with different acyl-chain lengths $[17,18]$. This model is formally a generalization of Eq. (1). The ten internal states and the associated degeneracies of each lipid species reflect the internal conformational statistics of long hydrocarbon chains. The interaction with integral membrane proteins is formally taken into account using the so-called hydrophobic-matching principle [14], which parametrizes the lipid-protein interaction in terms of a mismatch between the hydrophobic thickness of the lipid bilayer and the hydrophobic length of the protein. The protein, which is taken to have a hexagonal shape covering a number of sites on the triangular lipid lattice, is assumed to have two internal (conformational) states that are characterized by different values of the hydrophobic length. The two states of the protein, a ground state and an excited state, are furthermore characterized by two energy levels and an energy gap. The basic dynamics of the model is taken to be similar to that of the Ising model studied above. The proteins are activated on a time scale $\tau$; if the protein is in its ground state it will be excited (driven) to the excited state; if it is already in its excited state it will decay with the Boltzmann probability involving the energy gap as well as the change of interactions with the neighboring lipids.

Simulations of the steady-state properties of this driven membrane model have shown a behavior qualitatively similar to that described for the simple Ising model with active impurities. The results are described in a separate paper [16]. Given in Fig. 1(i) is an example of a microconfiguration for a steady-state situation of a binary lipid mixture with an annealed dispersion of active trans-membrane proteins subject to an external drive at conditions that in equilibrium would correspond to a phase-separated state. Similarly to the simple Ising model with active impurities, the drive on the proteins in the binary lipid mixture breaks down the macroscopically phase-separated state and induces a domain structure, or compartmentalized lateral membrane organization, characterized by a new length scale, which depends on the strength of the drive.

In conclusion, we have proposed a nonequilibrium model designed to describe the steady-state organization of multicomponent systems that are driven by input of energy from external sources via dissipation of energy into the mixed system through an annealed dispersion of active impurities. The models belong to the general class of driven diffusive systems [19] and are related to models with two thermodynamic temperatures as well as models where the ordering dynamics is coupled to chemical reactions. We have shown that the drive introduces a new length scale and therefore can be used as means of compartmentalizing multicomponent systems that otherwise under equilibrium conditions would be subject to macroscopic phase separation. A related use of chemical reactivity to compartmentalize polymeric materials was recently studied $[8,9,12]$. In contrast to these chemicalreaction systems or other systems in which the drive irreversibly produces interfacially active compounds, our model maintains the activity as a completely reversible process. In the case of lipid-proteins systems, such a principle of steadystate compartmentalization may be of substantial interest since it suggests a mechanism by which biological membranes can set up the compartments needed to steer enzymatic reactions on the membrane surface without having to cope with completely random diffusion of the reactants [20]. Since membrane proteins couple to membrane curvature [21], it would be of interest in this context to consider the possible coupling between protein activity and membrane curvature and its associated elastic modulii [22] (which we have left out of the present simple modeling). This could be of importance for mixed systems due to the existence of a local coupling between phase separation and curvature $[23,24]$ that will slow down the phase-separation process significantly [25]. Not all integral membrane proteins exhibit activity that can be sensed by the lipids. An example of an important membrane protein that does is rhodopsin [26], whose internal transitions, related to the visual process in the retina, are influenced by the hydrophobic matching to the lipids in the membrane.

This work was supported by the Danish Natural Science Research Council and the Danish Technical Research Council. O.G.M. was supported by the Canadian Institute for Advanced Research. 
[1] A. Bray, Adv. Phys. 43, 357 (1994).

[2] M. Laradji, H. Guo, M. Grant, and M. J. Zuckermann, Adv. Chem. Phys. 89, 159 (1995).

[3] L. Leibler, Makromol. Chem., Macromol. Symp. 16, 1 (1988).

[4] S. C. Glotzer, D. Stauffer, and N. Jan, Phys. Rev. Lett. 72, 4109 (1994).

[5] S. C. Glotzer and A. Coniglio, Phys. Rev. E 50, 4241 (1994).

[6] S. C. Glotzer, E. A. Di Marzio, and M. Muthukumar, Phys. Rev. Lett. 74, 2034 (1995).

[7] S. Toxvaerd, Phys. Rev. E 53, 3710 (1996).

[8] G. H. Fredrickson, Phys. Rev. Lett. 76, 3440 (1996).

[9] B. O'Shaughnessy and U. Sawhney, Phys. Rev. Lett. 76, 3444 (1996).

[10] J. J. Christensen, K. Elder, and H. C. Fogedby, Phys. Rev. E 54, R2212 (1996)

[11] D. Carati and R. Lefever, Phys. Rev. E 56, 3127 (1997).

[12] Q. Tran-Cong and A. Harada, Macromolecules 76, 1162 (1996).

[13] M. Bloom, E. Evans, and O. G. Mouritsen, Q. Rev. Biophys. 24, 293 (1991).
[14] O. G. Mouritsen and M. Bloom, Annu. Rev. Biophys. Biomol. Struct. 22, 145 (1993).

[15] K. Binder and D. Stauffer, Phys. Rev. Lett. 33, 1006 (1974).

[16] M. C. Sabra and O. G. Mouritsen, Biophys. J. 74, 745-752 (1998).

[17] J. Risbo, M. M. Sperotto, and O. G. Mouritsen, J. Chem. Phys. 103, 3643 (1995).

[18] K. Jørgensen and O. G. Mouritsen, Biophys. J. 65, 942 (1995).

[19] B. Schmittmann and R. K. P. Zia, in Phase Transitions and Critical Phenomena, edited by C. Domb and J. L. Lebowitz (Academic, London, 1995), Vol. 17, p. 13.

[20] E. C. C. Melo, I. M. Lourtie, M. B. Sankaram, T. E. Thompson, and W. L. C. Vaz, Biophys. J. 63, 1506 (1992).

[21] H. Aranda-Espinoza, A. Berman, N. Dan, P. Pincus, and S. Safran, Biophys. J. 71, 648 (1996).

[22] J. Prost and R. Bruinsma, Europhys. Lett. 33, 321 (1996).

[23] T. Taniguchi, Phys. Rev. Lett. 76, 4444 (1996).

[24] P. B. S. Kumar and M. Rao, Phys. Rev. Lett. 80, 2489 (1998).

[25] K. Jørgensen, A. Klinger, M. Braiman, and R. L. Biltonen, J. Chem. Phys. 100, 2766 (1996).

[26] M. Brown, Chem. Phys. Lipids 73, 159 (1994). 